\title{
Protective role of nuclear factor erythroid 2-related factor 2 in the hemorrhagic shock-induced inflammatory response
}

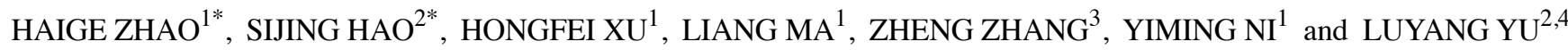 \\ ${ }^{1}$ Department of Cardiothoracic Surgery, The First Affiliated Hospital, School of Medicine, Zhejiang University, Hangzhou, \\ Zhejiang 310003; ${ }^{2}$ Institute of Genetics, College of Life Sciences, Zhejiang University, Hangzhou, Zhejiang 310058; \\ ${ }^{3}$ Eye Center of the Affiliated Second Hospital, School of Medicine, Zhejiang University, Hangzhou, Zhejiang 310009; \\ ${ }^{4}$ Research Center for Air Pollution and Health, Zhejiang University, Zhejiang University, Hangzhou, Zhejiang 310058, P.R. China
}

Received October 3, 2015; Accepted February 18, 2016

DOI: $10.3892 /$ ijmm.2016.2507

\begin{abstract}
Hemorrhagic shock (HS) following trauma or major surgery significantly contributes to mortality. However, the mechanisms through which HS activates the inflammatory response are not yet fully understood. Nuclear factor-erythroid 2 (NF-E2) p45-related factor-2 (Nrf2), a bZIP transcription factor, is a master regulator of robust cytoprotective defenses. The present study investigated the role of Nrf2 in the pathophysiology of HS. Nrf2 expression in peripheral leukocytes obtained from patients with surgery-associated hemorrhage subjected to resuscitation treatment (termed HS patients) or healthy donors was examined by RT-qPCR. A marked increase in Nrf2 expression was detected in the leukocytes obtained from the HS patients, which indicates a correlation between Nrf2 expression and the development of HS. Wild-type (WT; Nrf2 $2^{+/+}$) and Nrf2-deficient $\left[\mathrm{Nrf}^{-/-}\right.$or Nrf2-knockout (KO)] mice were subjected to surgery to induce HS. Systemic inflammation was significantly elevated in the Nrf2-KO mice compared with the WT mice following HS, as assessed by an increase in serum cytokine levels [interleukin (IL)-6, tumor necrosis factor (TNF)- $\alpha$ and IL-1 $\beta$ ], as well as high-mobility group box 1 protein (HMGB1) expression. The Nrf2-KO mice exhibited more severe lung and liver
\end{abstract}

Correspondence to: Dr Luyang Yu, Institute of Genetics, College of Life Sciences, Zhejiang University, Hangzhou, 866 Yuhangtang Road, Zhejiang 310058, P.R. China

E-mail: luyangyu@zju.edu.cn

Dr Yiming Ni, Department of Cardiothoracic Surgery, The First Affiliated Hospital, School of Medicine, Zhejiang University, 79 Qingchun Road, Hangzhou, Zhejiang 310003, P.R. China

E-mail: ni_yiming@hotmail.com

*Contributed equally

Key words: nuclear factor-erythroid 2 p45 subunit-related factor 2, hemorrhagic shock, ischemia/reperfusion, inflammatory response, pro-inflammatory cytokine injury following HS as evidenced by increased tissue damage, increased myeloperoxidase (MPO) activity and the increased production of pro-inflammatory cytokines. Additionally, Nrf2 deficiency augmented cytokine production induced by the exposure of peritoneal mouse macrophages to lipopolysaccharide (LPS) following HS. Taken together, these results suggest that Nrf2 is a critical host factor which limits immune dysregulation and organ injury following HS.

\section{Introduction}

Hemorrhagic shock (HS) significantly contributes to trauma-related mortality. HS and subsequent resuscitation are also responsible for the most common clinical complications in patients with traumatic injuries or who have undergone major surgery. Acute hypovolemia/hemodynamic disorders and global ischemia/reperfusion result in multiple organ failure mediated by priming the innate immune system (1-3). In this regard, an exaggerated inflammatory reaction prevails in distant vital organs, characterized by the activation and the infiltration of phagocytes (neutrophils and macrophages). It has been suggested that the liver and lungs are the most commonly affected organs following hemorrhage/resuscitation in clinical practice (4). As one of the first organs affected by HS, the liver suffers adenosine triphosphate (ATP) depletion due to ischemia, which results in the induction of inflammatory signaling and necrosis thereafter (5). On the other hand, the reperfusion phase in the liver consists of neutrophil activation mediated by inducible nitric oxide synthase, and ultimately leads to hepatocyte apoptosis $(6,7)$. By priming excess reactive oxygen species (ROS) production, ischemia/reperfusion activates neutrophils sequestered in the lung and alveolar macrophages, thus subsequently augmenting lung injury and dysfunction $(8,9)$. However, the precise mechanisms through which HS activates the inflammatory response have not yet been fully clarified.

Nuclear factor-erythroid 2 (NF-E2) p45-related factor-2 (Nrf2), an essential leucine zipper redox-susceptible transcription factor, is abundantly expressed in most tissues and is involved in regulating the induction of cytoprotective and antioxidant genes. Under conditions of oxidative stress, Nrf2 dissociates from its cytosolic inhibitor, Kelch-like 
ECH-associated protein 1 in the cytosol and translocates to the nucleus, where it promotes the production of antioxidants and transactivates related cytoprotective pathways (10). Accordingly, Nrf2 has been identified as a pivotal mediator in redox homeostasis and inflammatory disorders, including pulmonary fibrosis, asthma, cigarette smoke-induced emphysema, colonic inflammatory injury and experimental sepsis (11-15). Clinical studies have also concluded that there is a correlation between Nrf2 activity/signaling and the pathogenesis of inflammatory diseases, such as chronic obstructive pulmonary disease and chronic kidney disorder $(16,17)$.

Taking the above-mentioned findings into account, we hypothesized that Nrf2 exerts protective effects against inflammation-associated injury induced by HS, which may reveal a novel regulatory mechanism therein. To confirm this hypothesis, in this study, we examined the role of Nrf2 in the dysregulated inflammatory response following HS. We examined the correlation between $\mathrm{Nrf} 2$ expression and clinical HS. We also investigated the inflammatory response and subsequent injury due to HS in wild-type (WT; $\mathrm{Nrf}^{+/+}$) and Nrf2-deficient [Nrf2 ${ }^{-/-}$or Nrf2-knockout $\left.(\mathrm{KO})\right]$ mice in vivo and ex vivo.

\section{Materials and methods}

Clinical specimens, processing and RT-qPCR. The protocols for blood collection and analysis were approved by the Ethics Review Committee of Zhejiang University School of Medicine (Hangzhou, China). Additionally, written informed consent was obtained from all patients prior to blood collection. Whole blood was obtained from 6 blood donors without any disease or from 6 patients with surgery-associated hemorrhage subjected to resuscitation treatment (termed HS patients), of which 3 were undergoing hepatectomy, 2 splenectomy and 1 abdominal aorta replacement. All 6 patients suffered blood loss $>15 \%$ of total blood volume with the duration of $\mathrm{HS}$ varying from 8-30 min. All the surgeries were performed at the First Affiliated Hospital of Zhejiang University School of Medicine. We collected the blood samples at $1 \mathrm{~h}$ and 5 days post-operation. Briefly, blood samples were obtained from a forearm vein and coded without patient identifiers. All specimens were collected in Vacutainer tubes containing the anticoagulant, ethylenediamine tetraacetic acid (EDTA; Kang Shi Medical Equipment Co., Ltd., Hangzhou, China) and were processed within $2 \mathrm{~h}$. During sample collection and processing, whole blood specimens were stored at $4^{\circ} \mathrm{C}$. For blood specimen processing, whole blood was treated with $5 \mathrm{X}$ BL solution [from a Blood gDNA mini kit (Biomiga, San Diego, CA, USA)] for erythrocyte depletion and then centrifuged at 3,000 $\mathrm{x} g$ for 10 min at $4^{\circ} \mathrm{C}$ in order to collect the leukocytes.

Total RNA was extracted using the Total RNA kit I (Omega Bio-Tek, Inc., Doraville, GA, USA) after the cells were lysed using $500 \mu \mathrm{l}$ TRIzol reagent (Invitrogen, Carlsbad, CA, USA). First-strand cDNA was synthesized from $200 \mathrm{ng}$ of total mRNA using the ReverTra Ace qPCR RT Master Mix with a gDNA Remover kit (Toyobo, Osaka, Japan). Reverse transcription-quantitative polymerase chain reaction (RT-qPCR) was performed using the All-in-One qPCR Mix kit (GeneCopoeia, Rockville, MD, USA) and analyzed using the $\Delta \Delta \mathrm{Ct}$ method on a CFX Connect Real-Time PCR system (Bio-Rad, Hercules,
CA, USA). The reaction was initiated at $95^{\circ} \mathrm{C}$ for $10 \mathrm{~min}$, followed by 40 cycles of denaturation at $95^{\circ} \mathrm{C}$ for $15 \mathrm{sec}$, annealing at $55^{\circ} \mathrm{C}$ for $20 \mathrm{sec}$ and extension at $72^{\circ} \mathrm{C}$ for $20 \mathrm{sec}$. A primer pair for the detection of human GAPDH was used as an internal control. The primers used for RT-qPCR in the present study were as follows: Nrf2 (human) sense, 5'-TCAGCGACGGAAA GAGTATGA-3' and antisense, 5'-CCACTGGTTTCTGACT GGATGT-3'; and GAPDH (human) sense, 5'-CATTGCCCTC AACGACCACTTTGT-3' and antisense, 5'-TCTCTCTCTT CCTCTTGTGCTCTTGC-3'.

Mice. All animal experimental protocols were performed in accordance with the Policy and Procedures Manual of Zhejiang University Animal Care and Use Committee (Hangzhou, China). Nrf2-KO mice were generated as described in our previous study (18). The Nrf2-KO mice in the current study were provided by Dr Rajesh K. Thimmulappa and Dr Shyam Biswal who obtained the mice as a gift from a Japanese group (18). Briefly, the b-Zip region of mouse nrf2 cDNA was replaced with SV40 nuclear localization signal- $\beta$-galactosidase (lacZ) gene by homologous recombination. The linearized targeting construct was electroporated into embryonic stem cells (ES) and positive colonies were identified by PCR and Southern blot analysis. Positive ES colonies were then introduced into C57BL/6J blastocysts by microinjection to obtaine chimeric mice, which were mated with ICR females and BALB/cA females for germline transmission. Afterwards, F1 and F2 offspring were identified by genotyping. Nrf2-KO mice were then backcrossed to the C57BL/6 strain over 12 generations to obtain WT mice of the same origin.

Induction of $H S$. Twenty mice were subjected to hemorrhage and rescusitation (termed the HS group) and another 20 mice were subjected to sham-operation according to previously described protocols (19). Briefly, the mice were anesthetized with isoflurane (Minrad, Inc., Orchard Park, NY, USA) after being fasted overnight with only water ad libitum. Polyethylene-10 tubing (BD Biosciences, San Jose, CA, USA) was placed within both femoral arteries and the right femoral vein. Upon awakening, the mice were bled rapidly through the other arterial catheter to a mean arterial blood pressure of $35 \pm 5 \mathrm{mmHg}$ within $10 \mathrm{~min}$, which was then maintained for the remaining $90 \mathrm{~min}$. At the end of the above-mentioned period, the mice were resuscitated via the venous line using a volume of Ringer's lactate solution equal to 4 times the volume of blood lost. Following blood vessel ligation, all catheters were removed and the incisions were closed with sutures. The mice in the sham-operated group were subjected to the same surgical procedures as those in the HS group, but were not subjected to hemorrhage or resuscitation.

Histological analysis. To perform histological analysis, the livers and lungs were harvested from the mice as indicated above. Briefly, the mice were anaesthetized with ketamine and xylazine and sacrificed by cervical dislocation $24 \mathrm{~h}$ following HS or sham-operation. After removing the hair, the abdomens and chest of mice were cut vertically and opened, revealing the liver and lungs. Half of the two tissues were then isolated, respectively. The tissues were then fixed in $4 \%$ paraformaldehyde and embedded in paraffin. The paraffin blocks were then 
sectioned at 5- $\mu \mathrm{m}$ thick using a microtome (Leica Biosystems, Heidelberger, Germany). After floating on a $40^{\circ} \mathrm{C}$ water bath containing distilled water, the sections were transferred onto the surface of clean glass slides, which were placed in $37^{\circ} \mathrm{C}$ oven to dry overnight and stored in light-proof boxes until ready for use. Sections (5- $\mu \mathrm{m}$-thick) were prepared and stained with hematoxylin and eosin. Two independent investigators performed the quantitative analysis of liver and lung injuries by assessing the histological scores. The extent of lung injury was determined by grading 4 histological findings: congestion, edema, inflammation and hemorrhage. The degree of lung injury was scored on a scale of $0-4$ ( 0 , normal; 1 , mild; 2 , moderate; 3 , severe; and 4 , very severe) for each feature, with a cumulative maximum score of 16. For liver injury, all sections were examined for the following 6 parameters: cytoplasmic color fading, vacuolization, nuclear condensation, nuclear fragmentation, nuclear fading and erythrocyte stasis. Each parameter was scored according to the percentage of cells showing the particular parameter per 10 microscopic fields: $0,0 \%, 1,0-10 \% ; 2,10-50 \%$; and 3, 50-100\%. The histological scores are reported as the sum of the individual values.

Measurement of cytokine levels by ELISA. The levels of interleukin (IL)- $1 \beta$ and tumor necrosis factor (TNF)- $\alpha$ in plasma and protein lysis were measured using ELISA kits (eBioscience, San Diego, CA, USA) according to the manufacturer's instructions. The IL-6 levels were also measured using an ELISA kit (R\&D Systems, Minneapolis, MN, USA). The plasma was obtained with the following protocol: $2 \mathrm{~h}$ following HS or sham-operation, the mice were anaesthetized with ketamine and xylazine and fixed in a supine position. The mouse left chest between the 3 to 4 intercostal space was felt using an index finger of the left hand to feel the heartbeat. Afterwards, a $23 \mathrm{G}$ needle of a syringe was carefully punctured into the mouse chest cavity until it entered the heart. When the heart was punctured, the blood rushed into the syringe automatically, and $0.5-0.6 \mathrm{ml}$ blood was collected in vacutainer tubes containing the anticoagulant, EDTA. The plasma was obtained by removing blood cells and platelets by centrifugation for $15 \mathrm{~min}$ at 2,000 x g. Protein lysis of the lung and liver tissue was carried out as follows: the isolated liver and lung tissues were placed in liquid nitrogen and then smashed with a wooden hammer. Afterwards, the tissue powders were resuspended in RIPA buffer (1\% Triton X-100, 1\% deoxycholate and $0.1 \%$ SDS), which was centrifuged at $15,000 \mathrm{x}$ g for $15 \mathrm{~min}$ at $4^{\circ} \mathrm{C}$. The supernatant was carefully isolated as tissue protein lysis.

Immunoblot analysis. Protein was extracted from the serum in lysis buffer (1\% NP-40, 0.5\% sodium deoxycholate, $0.1 \%$ SDS, $5 \mathrm{mM}$ EDTA, $1 \mathrm{mM}$ sodium orthovanadate, $0.1 \%$ aprotinin and $1 \mathrm{mM}$ PMSF) and boiled in SDS sample buffer for 5 min. Equal amounts of protein per sample were separated by SDS-PAGE and transferred electrophoretically onto a polyvinylidene fluoride membrane (Bio-Rad Laboratories). After blocking in 5\% milk, the membrane was incubated with a primary antibody followed by an HRP-conjugated secondary antibodies (115-475-062, goat anti-mouse IgG and 111-475-003, goat anti-rabbit IgG; Jackson ImmunoResearch, West Grove, PA, USA). Chemiluminescence was then detected using an ECL kit according to the manufacturer's instructions (Amersham Life Science, Arlington Heights, IL, USA). The primary antibodies, anti-high-mobility group box 1 protein (HMGB1) (ab18256, rabbit anti-mouse/human, polyclonal) and anti- $\beta$-actin (ab8226, mouse anti-mouse/human, monoclonal), were purchased from Abcam (Cambridge, UK).

Myeloperoxidase (MPO) activity assay. MPO activity was assayed using techniques described previously (20). Briefly, the tissue samples were sonicated using an Ultrasonic Cell Disrupter (Kontes, Vineland, NJ, USA) with 10 intervals for 9 times, then incubated in a $60^{\circ} \mathrm{C}$ water bath for $1.5 \mathrm{~h}$, and centrifuged at $10,500 \mathrm{x} \mathrm{g}$ for $15 \mathrm{~min}$. For each assay, $100 \mu \mathrm{l}$ of collected supernatant were mixed with $2.9 \mathrm{ml}$ assay solution (50 mM potassium phosphate buffer, containing $5 \times 10^{-4} \% \mathrm{H}_{2} \mathrm{O}_{2}$ and $0.167 \mathrm{mg} / \mathrm{ml} o$-dianisidine, $\mathrm{pH}$ 6.0). MPO activity was then determined by measuring the absorbance of $460 \mathrm{~nm}$ visible light using a spectrophotometer (Beckman DU7; Beckman Coulter, Brea, CA, USA) and finally calculated in units per microgram of wet tissue.

Culture and treatment of mouse peritoneal macrophages. To isolate peritoneal macrophages, the mice were injected with $2 \mathrm{ml} \mathrm{4 \%}$ thioglycollate broth (Sigma-Aldrich, St. Louis, MO, USA) and incubated for 3 days prior to being subjected to HS or sham operation. At $24 \mathrm{~h}$ after resuscitation, they were anesthetized and sacrificed by cervical dislocation. For the harvesting of peritoneal macrophages $5 \mathrm{ml}$ ice-cold PBS was injected into the peritoneal cavity and peritoneal exudates were aspirated after 2 min under sterile conditions. The isolated peritoneal macrophages were cultured at a density of $2.5 \times 10^{5}$ cells $/ \mathrm{ml}$ in RPMI-1640 medium (Gibco-Invitrogen Cell Culture, Carlsbad, CA, USA) containing $10 \%$ fetal bovine serum under $5 \% \mathrm{CO}_{2}$ at $37^{\circ} \mathrm{C}$. The medium was replaced after $1 \mathrm{~h}$ in order to remove the unattached cells. Lipopolysaccharide (LPS; $100 \mathrm{ng} / \mathrm{ml}$ ) was then added to the medium. The supernatant was collected after $6 \mathrm{~h}$ for measuring the concentration of IL-1 $\beta$, IL- 6 and TNF- $\alpha$.

Statistical analysis. All data were analyzed using the unpaired Student's t-test or one-way ANOVA. A p-value $<0.05$ was considered to indicate a statistically significant difference.

\section{Results}

Marked induction of Nrf2 expression in leukocytes is associated with clinical HS. Nrf2 expression in leukocytes has been described in a few in vitro studies $(21,22)$. In this study, to identify the potential role of Nrf2 in the HS-induced inflammatory response, the expression pattern of Nrf2 in leukocytes was investigated. To this end, whole blood samples were collected from patients with surgery-associated hemorrhage subjected to resuscitation treatment, or from healthy donors. Isolated leukocytes from these clinical samples were then subjected to RT-qPCR for Nrf2. A normal expression of Nrf2 was detected in the whole blood samples from the healthy donors, whereas a significant increase in the Nrf2 expression levels was observed in the samples collected from the HS patients (Fig. 1). Furthermore, the trend in the induction of Nrf2 expression was synchronized with the duration of HS (Fig. 1). Thus, these 


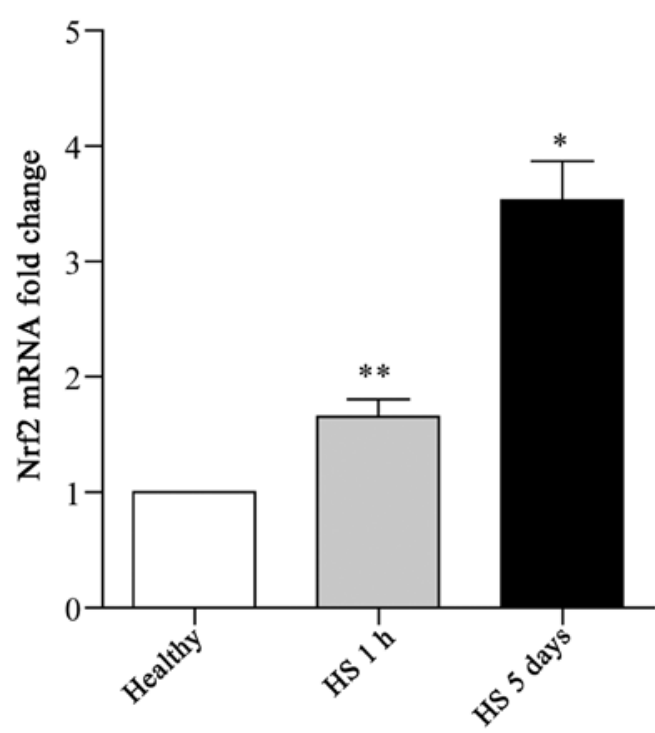

Figure 1. Marked induction of nuclear factor erythroid 2-related factor 2 (Nrf2) in leukocytes isolated from patients with hemorrhagic shock (HS). Whole blood samples were collected from healthy donors and patients with surgicalassociated hemorrhage subjected to resuscitation treatment after $1 \mathrm{~h}$ or 5 days. White blood cells were subsequently isolated from these clinical samples and the transcript levels of Nrf2 were then quantified by RT-qPCR. Data shown are the means \pm SEM from 6 samples per group. * $\mathrm{p}<0.05 \mathrm{vs}$. healthy donor samples, ${ }^{* *}$ p $<0.001$ vs. healthy donor samples.

findings indicate that there is a close correlation between Nrf2 expression and the development of HS.

Nrf2 deficiency aggravates lung and liver injury in a mouse model of HS. In order to further explore the function of Nrf2 in the pathological setting of HS, we established a mouse model of HS, which mimicked clinical HS, as indicated in the Materials and methods. More severe lung and liver injury was observed in the Nrf2-KO mice following HS. Histological analysis revealed more interstitial edema, alveolar wall thickening and the interstitial infiltration of leukocytes, as well as alveolar hemorrhage in the lungs of the Nrf2-KO mice compared with the WT group (Fig. 2B, with the histological score shown in Fig. 2D). Similarly, the livers of the Nrf2-KO mice subjected to HS had far more interstitial edema, leukocyte infiltration and interstitial hemorrhage, as well as more severe hepatocellular injury with necrotic areas (Fig. 2A, with the histological score shown in Fig. 2C). On the other hand, the extent of organ injury was evaluated by determining the levels of pro-inflammatory cytokines in the organ tissues. We measured the levels of these cytokines in homogenized tissue protein by ELISA. In the lungs and livers of all the mice, the levels of IL- 6 and TNF- $\alpha$ were significantly increased following HS; however, the Nrf2-KO mice exhibited significantly higher levels of these cytokines compared with the WT mice (Fig. 3A, B, E and F). The Nrf2-KO mice exhibited much higher expression levels of IL-1 $\beta$ in the lungs compared with the WT mice following HS, whereas there was no significant difference observed in the IL-1 $\beta$ expression levels in the livers of the Nrf2-KO and WT mice (Fig. 3C and D). The accumulation of neutrophils in the lung and liver tissues was assessed by measuring MPO activity, which is also indicative of the severity of inflamma- tion in the organs. Following HS, an increased MPO activity in the lungs and livers was detected in both the WT and Nrf2-KO mice; however, the Nrf2-KO mice had a significantly higher MPO activity than the WT mice in both organs (Fig. 4). Taken together, these findings indicate that Nrf2 deficiency induces a more severe inflammatory response in the vital organs examined (lungs and liver) following HS.

Nrf2 deficiency exacerbates systemic inflammation induced by $H S$. In $\alpha$ previous study, Nrf 2 was shown to be a critical transcription factor in lethal septic shock (15). In this study, to determine the role of Nrf2 in HS, we compared the severity of systemic inflammation in the WT and Nrf2-KO mice. As IL-6, IL-1 $\beta$ and TNF- $\alpha$ are well established as key cytokines involved in the mediation of inflammation and associated mortality (23), the plasma concentrations of these cytokines were thereby examined following HS. The results of ELISA revealed an increasing trend in the levels of IL-6, IL-1 $\beta$, and TNF- $\alpha 2 \mathrm{~h}$ following HS. However, the levels of all 3 cytokines were significantly higher in the Nrf2-KO mice compared with the WT mice (Fig. 5). On the other hand, HMGB1, a cytokine and alarmin molecule, has been characterized as a crucial early mediator of inflammation after HS and organ ischemia/ reperfusion (24). Thus, in the present study, we also examined the expression of HMGB1 in mouse plasma following HS and a marked induction in HMGB1 expression in the Nrf2-KO group was detected by immunoblot analysis (Fig. 6). These data suggest that Nrf2 is a negative mediator for modulating systemic inflammation in HS.

Nrf2 deficiency augments cytokine production induced by LPS in peritoneal macrophages following HS. As the major component of innate immunity, macrophages play an essential role in the inflammatory response (23). In this study, to evaluate the potential anti-inflammatory effects of Nrf2 in HS, mouse peritoneal macrophages were harvested $24 \mathrm{~h}$ after resuscitation and then cultured with LPS for a further $6 \mathrm{~h}$. The levels of cytokine production in the supernatant were subsequently determined using ELISA. The results revealed an undetectable level of IL-1 $\beta$ (data not shown), whereas there was an abundant level of IL-6 and TNF- $\alpha$ in the peritoneal macrophages. At the basal level, the macrophages produced similarly low levels of IL- 6 and TNF- $\alpha$ in the cells from both the WT and Nrf2-KO mice. Following exposure to LPS, the level of IL-6 was significantly higher in the macrophages from the Nrf2-KO mice compared with those from the WT mice, even in the sham-operated groups. However, when making comparisons between the same mouse strains in the 2 groups (HS group and sham-operated group), we found that IL-6 production was significantly increased only in the macrophages from the Nrf2-KO mice with HS compared to those from the Nrf2-KO mice in the sham-operated group; there were no significant differences observed in the IL-6 levels between the WT mice in the HS and sham-operated groups (Fig. 7A). Notably, a decrease in TNF- $\alpha$ levels induced by LPS was detected in the macrophages from the WT mice subjected to HS, whereas there was an increase in these levels in the cells from the Nrf2-KO mice (Fig. 7B). Thus, Nrf2 deficiency resulted in the increased production of inflammatory cytokines in the LPS-exposed macrophages from mice subjected to HS. 
A
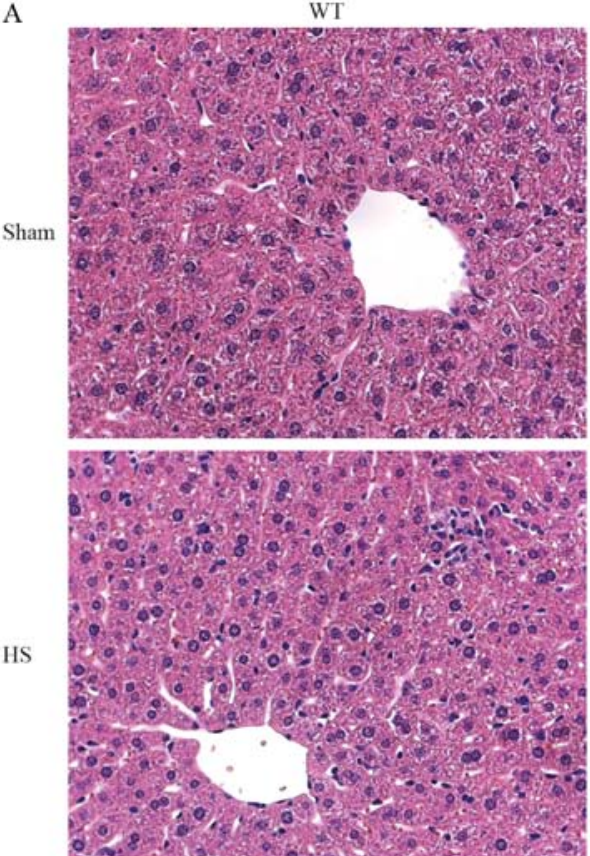

Nrf2-KO
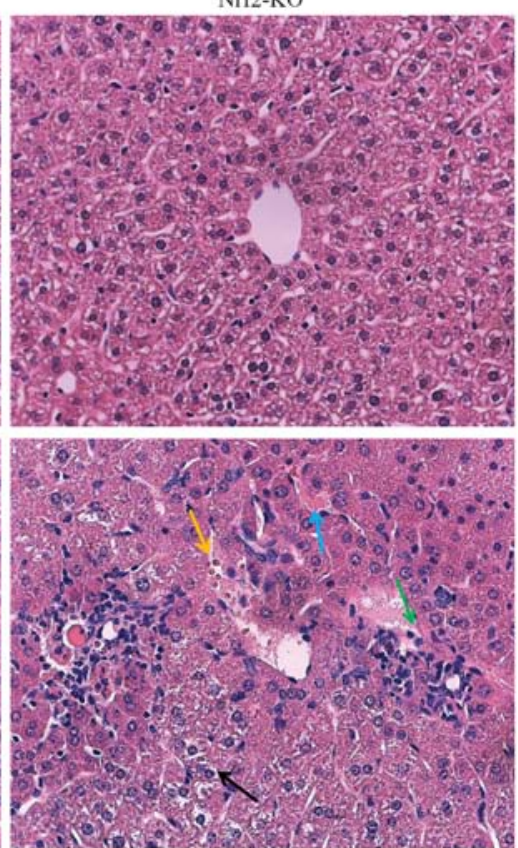

B
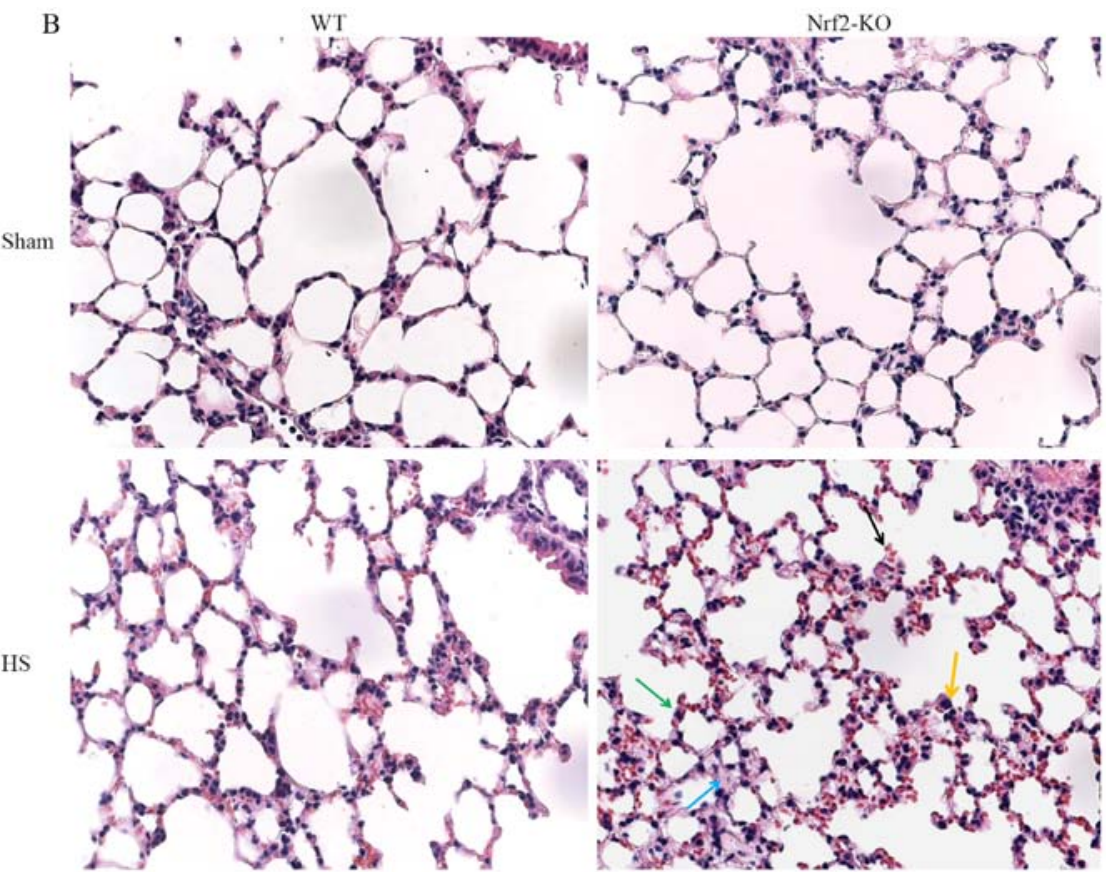

C

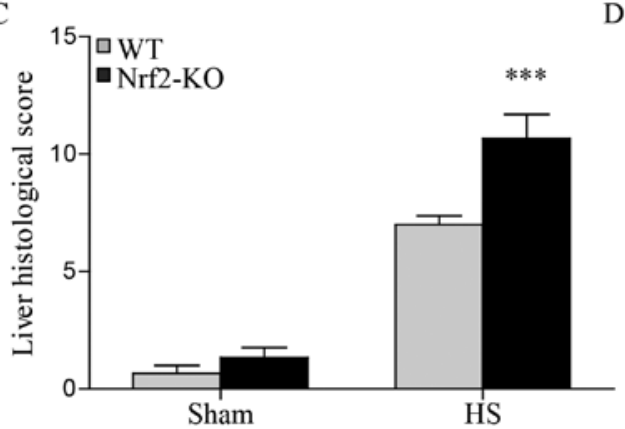

$\mathrm{D}$

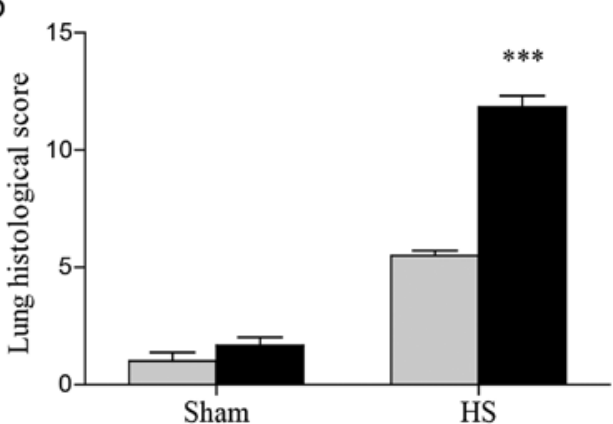

Figure 2. Nuclear factor erythroid 2-related factor 2 (Nrf2)-KO mice exhibit more severe liver and lung injury following hemorrhagic shock (HS). Lungs and livers of wild-type (WT) and Nrf2-KO mice were harvested $24 \mathrm{~h}$ after HS. Tissues were then processed for paraffin sectioning and hematoxylin and eosin staining. Representative images of liver sections from the 2 experimental groups are presented in (A). Photomicrographs were captured using a Zeiss microscope with a 40X objective. The blue arrow indicates interstitial edema, the green arrow indicates leukocyte infiltration, the yellow arrow indicates interstitial hemorrhage and the black arrow indicates hepatocellular injury with necrotic areas in the liver. Representative images of lung sections from 2 experimental groups are demonstrated in (B). Photomicrographs were obtained with a Zeiss microscope with a 40X objective. The blue arrow indicates interstitial edema, the green arrow indicates alveolar wall thickening, the yellow arrow indicates interstitial infiltration of leukocytes and the black arrow indicates alveolar hemorrhage in the lungs. Quantitative assessments of (C) liver or (D) lung injury are presented. Data shown are the means \pm SEM ( $\mathrm{n} \geq 5$ samples per group). ${ }^{* * *} \mathrm{p}<0.001 \mathrm{vs.} \mathrm{WT} \mathrm{mice.} \mathrm{Sham,} \mathrm{sham-operated} \mathrm{group.}$ 
A

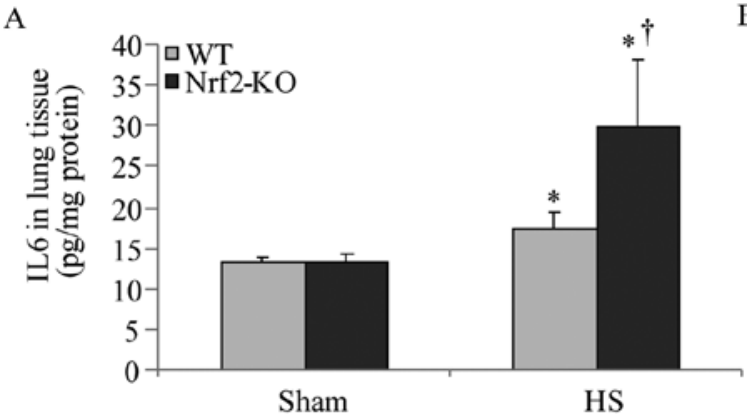

C

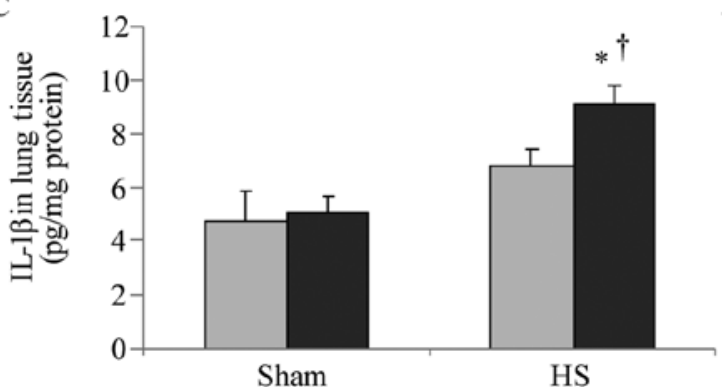

B

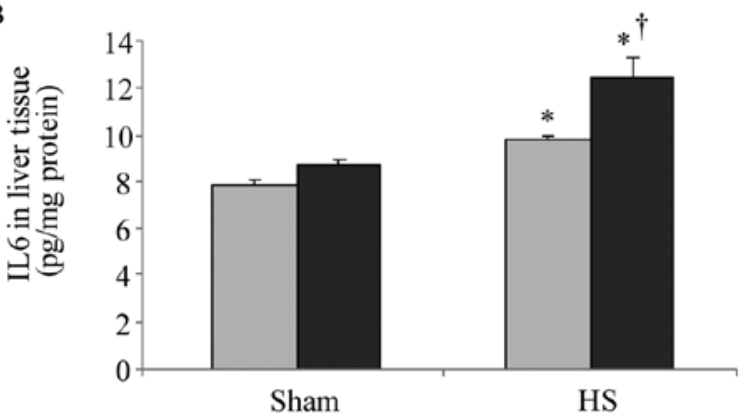

D

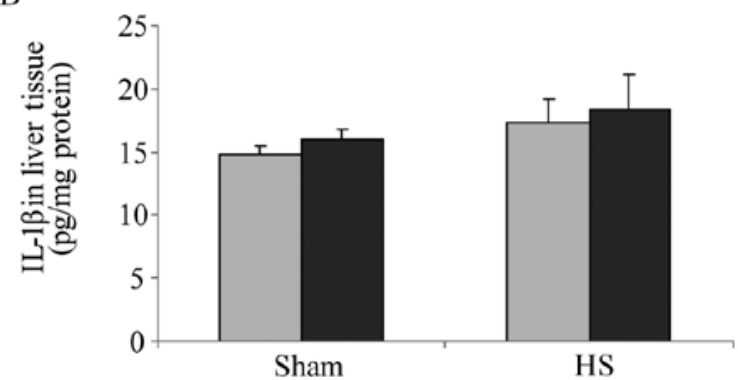

E

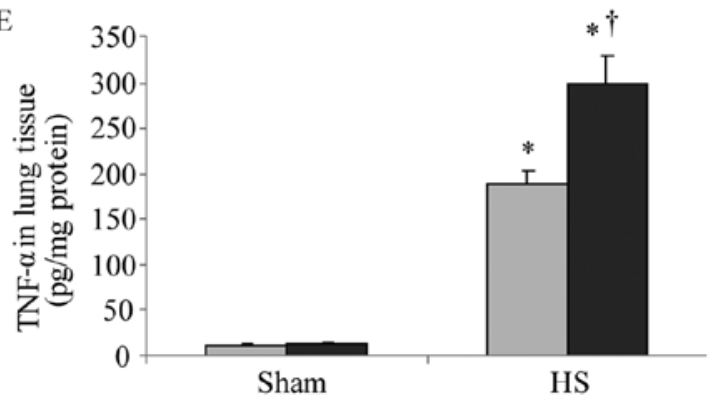

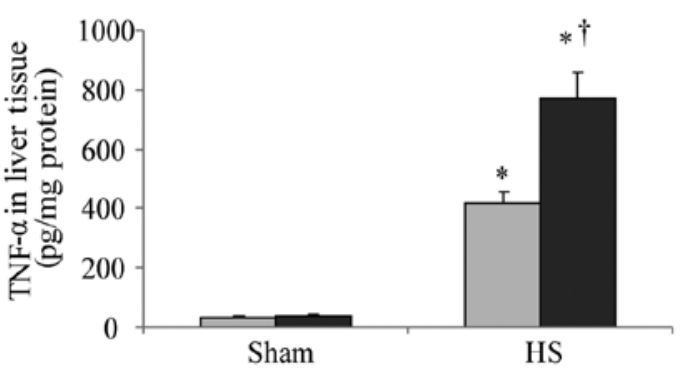

Figure 3. Increased production of pro-inflammatory cytokines in lungs and livers of nuclear factor erythroid 2-related factor 2 (Nrf2)-KO mice subjected to hemorrhagic shock (HS). Lungs and livers of wild-type (WT) and Nrf2-KO mice were harvested $24 \mathrm{~h}$ after HS. The concentrations of (A and B) interleukin (IL)-6, (C and D) IL-1 $\beta$ and (E and F) tumor necrosis factor (TNF)- $\alpha$ were assessed by ELISA. Results are expressed as pg/mg tissue protein. Data represent the mean cytokine levels and are shown as the means \pm SEM ( $\mathrm{n} \geq 4$ samples per group). ${ }^{*} \mathrm{p}<0.05$ vs. sham-operated group (sham); ${ }^{\dagger} \mathrm{p}<0.05 \mathrm{vs.} \mathrm{WT} \mathrm{mice.}$

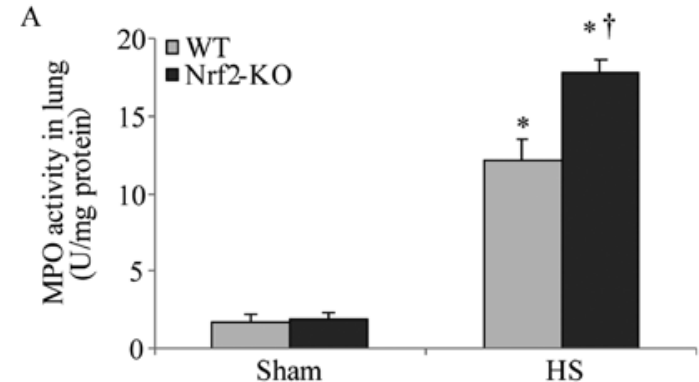

B

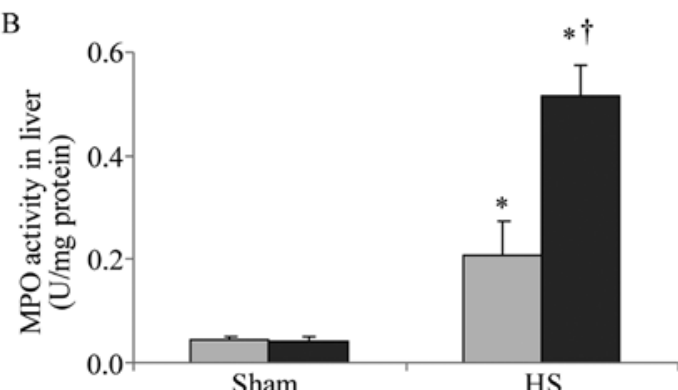

Figure 4. Higher myeloperoxidase (MPO) activity in lungs and livers of nuclear factor erythroid 2-related factor 2 (Nrf2)-KO mice subjected to hemorrhagic shock (HS). MPO activity was assessed as enzyme activity in tissue protein of (A) lung and (B) liver samples $2 \mathrm{~h}$ after HS. Data shown are the means \pm SEM ( $\mathrm{n} \geq 4$ samples per group). ${ }^{*} \mathrm{p}<0.05$ vs. sham-operated group (sham); ${ }^{\dagger} \mathrm{p}<0.05$ vs. WT mice.

\section{Discussion}

HS is a medical emergency, characterized by the sudden major loss of intravascular volume, which may cause death due to massive blood loss. Even though fluid resuscitation may restore circulatory failure, it also increases the severity of ischemia/reperfusion injury, which induces multiple organ dysfunction and progressive multiple organ failure $(25,26)$. Under these circumstances, overwhelming oxidative stress is the major consideration, resulting from failed mitochondrial aerobic metabolism and excessive NADPH oxidase-dependent ROS generation in the cytoplasm (27-29). However, the precise regulatory mechanisms responsible for oxidative stress-mediated HS injury remain largely unknown. In the present study, clinical analysis demonstrated significantly increased Nrf2 levels in the patients with surgery-associated hemorrhage subjected to resuscitation treatment, and the Nrf2 levels increased in a time-dependent manner in these patients. These results suggest that there is a correlation between Nrf2 expression and the development of HS. Based on these findings, as well as the results of a previous study investigating the role of Nrf2 in the pathogenesis of sepsis (15), we hypothesized that $\mathrm{Nrf2}$, the critical transcriptional factor mediating antioxidant 
A

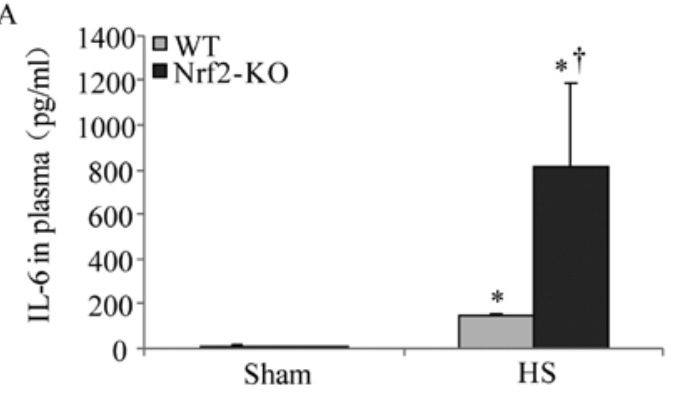

B

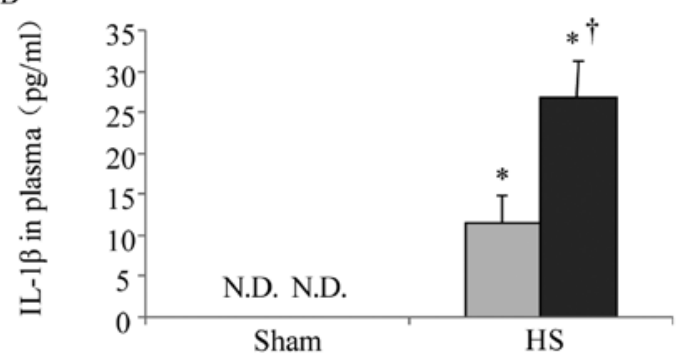

$\mathrm{C}$

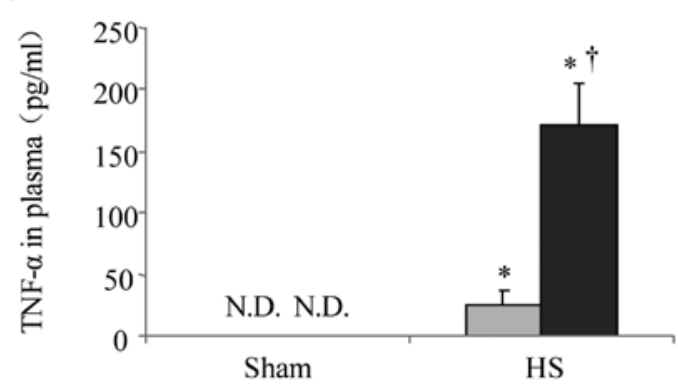

Figure 5. Nuclear factor erythroid 2-related factor 2 (Nrf2)-KO mice exhibit increased systemic inflammation following hemorrhagic shock (HS). Plasma from wild-type (WT) and Nrf2-KO mice was collected $2 \mathrm{~h}$ after HS. (A) Interleukin (IL)-6, (B) IL-1 $\beta$ and (C) tumor necrosis factor (TNF)- $\alpha$ plasma levels were determined by ELISA. The results represent mean cytokine levels shown as the means \pm SEM ( $\mathrm{n} \geq 4$ samples per group). N.D., not detected. ${ }^{*} \mathrm{p}<0.05$ vs. sham-operated group (sham), ${ }^{\dagger} \mathrm{p}<0.05$ vs. WT mice.

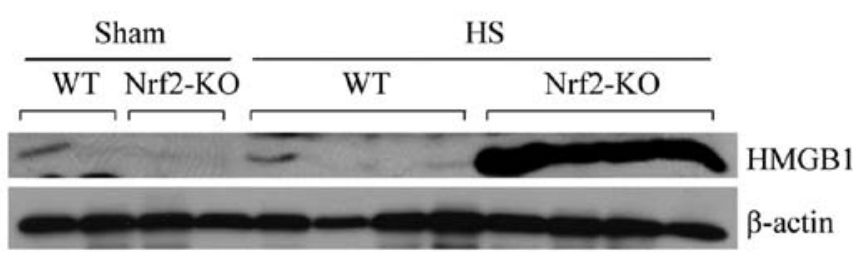

Figure 6. Increased expression of high-mobility group box 1 protein (HMGB1) in nuclear factor erythroid 2-related factor 2 (Nrf2)-KO mice subjected to hemorrhagic shock (HS). Plasma from wild-type (WT) and Nrf2-KO mice was collected $24 \mathrm{~h}$ after HS. HMGB1 expression was determined by immunoblot analysis. Each band represents levels of HMGB1 in $3 \mu 1$ plasma of an individual mouse.

signaling, plays an important role in inflammatory injury following HS. To this end, Nrf2-KO mice with WT mice being used as controls were employed in order to establish an animal model of HS. Histological analysis revealed that Nrf2 deficiency resulted in more severe liver and lung injury; the liver and lungs are major organs that are very responsive to ischemia/reperfusion during HS $(30,31)$. Thus, these findings indicate that Nrf2 potentially exerts protective effects against organ injury following HS.
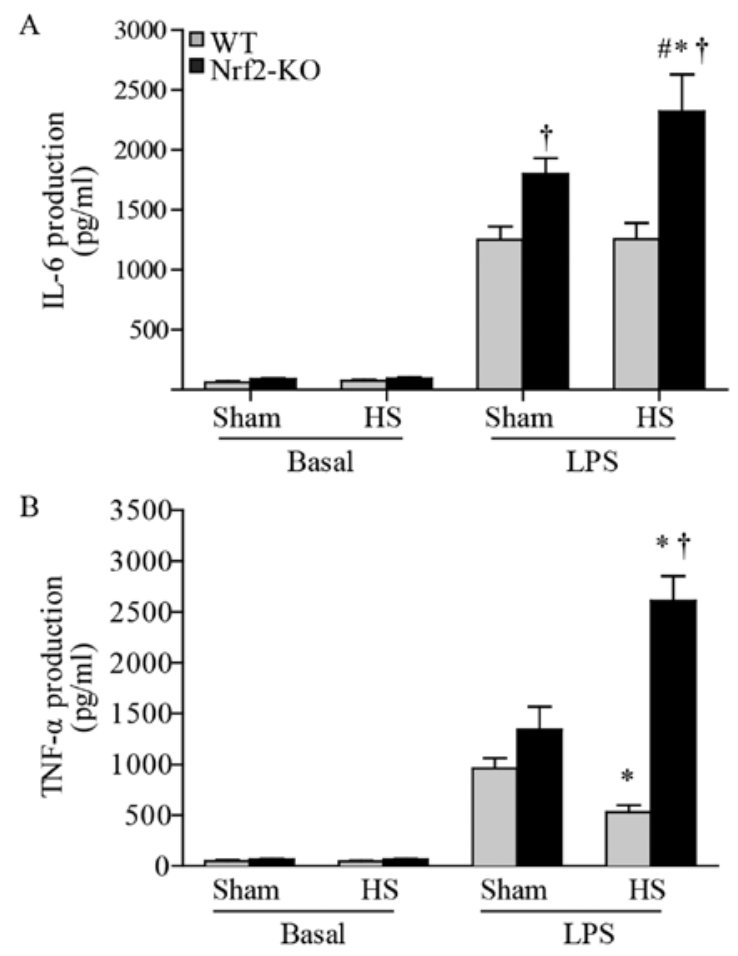

Figure 7. Nuclear factor erythroid 2-related factor 2 (Nrf2) deficiency augments the lipopolysaccharide (LPS)-induced production of cytokines by peritoneal macrophages following hemorrhagic shock (HS). Peritoneal macrophages were harvested at $24 \mathrm{~h}$ after $\mathrm{HS}$ and then treated with LPS $(100 \mathrm{ng} / \mathrm{ml})$. Concentrations of (A) interleukin (IL)-6, (B) tumor necrosis factor (TNF)- $\alpha$ with or without LPS treatment were assessed by ELISA. IL- $1 \beta$ was undetectable in the supernatant. Data shown are the means \pm SEM ( $\mathrm{n} \geq 4$ samples per group). ${ }^{\mathrm{p}}<0.05 \mathrm{vs}$. sham-operated group (sham), ${ }^{\dagger} \mathrm{p}<0.05$ vs. wild-type mice (WT), ${ }^{\#} \mathrm{p}<0.05$ vs Nrf2-KO mice in the sham-operated group.

It is well known that the ROS-mediated activation of the innate immune response is the dominant modulator of multiple organ failure and even mortality due to HS $(32,33)$. Excess levels of intracellular and extracellular ROS induce the hyper-activation of innate immune cells $(34,35)$. In this study, to determine whether Nrf2 is involved in this process, we further investigated the activation of neutrophils and macrophages in Nrf2-KO mice compared with WT mice; both were subjected to HS. Nrf2 deficiency enhanced MPO-1labeled neutrophil activation in vivo, as well as LPS-induced macrophage activation ex vivo. Additionally, the production of classical innate inflammatory cytokines, namely IL-6, IL-1 $\beta$ and TNF- $\alpha$, was highly elevated in the Nrf2-KO group, both in the blood and in local organs. Under these circumstances, neutrophils and macrophages are the major sources of these pro-inflammatory cytokines, as we confirmed. Such results further support the negative effects of Nrf2 on the augmentation of the innate immune response. On the other hand, the development of systemic inflammatory response syndrome during HS is characterized by the excessive production of these pro-inflammatory cytokines, which results in end-organ injury. Animal and clinical studies have demonstrated the key pathological event of the imbalance between pro-inflammatory and anti-inflammatory cytokine production for the progression of organ failure in HS $(36,37)$. Of note, the downregulation of proinflammatory cytokines in the early pathological process of HS 
is of utmost importance. Concerning our results, the constitutive protective function of Nrf2 in inflammation following HS may therefore be attributed to the suppression of pro-inflammatory cytokine production at the initial stage of the immune response.

Notably, the expression of circulatory HMGB1 was markedly upregulated in the absence of Nrf2 in mice. HMGB1 was originally identified as a nuclear DNA-binding protein and subsequently as a novel late-acting inflammatory cytokine, which mediates lethality in sepsis $(38,39)$. HMGB1 has been identified as an inducible alarmin molecule for cell injury at the early stages of inflammation. During HS ischemia/reperfusion, HMGB1 has been shown to be highly activated and released in neutrophils and macrophages, acting as an inflammatory mediator responsible for acute lung and liver injury $(40,41)$. In a clinical setting, increased concentrations of HMGB1 have been detected in the serum of patients with HS and its neutralization may improve related clinical outcomes $(42,43)$. Therefore, the inhibitory effects of Nrf2 on HMGB1 expression may contribute to another regulatory mechanism of Nrf2 in organ injury following $\mathrm{HS}$.

In conclusion, the present study demonstrated that $\mathrm{Nrf} 2$ played a negative role in the activation of the innate immune response during HS by targeting pro-inflammatory cytokine production and HMGB1 expression, thereby inhibiting inflammation-associated organ injury. Thus, Nrf2 is an important regulator in protecting major organs from ischemia/reperfusion damage in HS. The modulation of systemic Nrf2 expression and activity may represent a novel strategy for the treatment of HS-related disease.

\section{Acknowledgements}

The present study was supported by the National Natural Science Foundation of China (grant nos. 81000138, 81270357, 81422005 and 31470057), the Zhejiang Provincial Natural Science Foundation of China (grant no. LR14H020002), the Medical Science Research Foundation of Zhejiang Province (grant no. 2012KYB102), the Traditional Chinese Medicine Foundation of Zhejiang Province (grant no. 2012ZA083) and the Fundamental Research Funds for the Central Universities. We would also like to thank Dr Rajesh K. Thimmulappa and Dr Shyam Biswal (Johns Hopkins University) for providing extensive assistance with the current study.

\section{References}

1. Jarrar D, Chaudry IH and Wang P: Organ dysfunction following hemorrhage and sepsis: mechanisms and therapeutic approaches (Review). Int J Mol Med 4: 575-583, 1999.

2. McGhan LJ and Jaroszewski DE: The role of toll-like receptor-4 in the development of multi-organ failure following traumatic haemorrhagic shock and resuscitation. Injury 43: 129-136, 2012.

3. Visser T, Pillay J, Koenderman L and Leenen LP: Postinjury immune monitoring: can multiple organ failure be predicted? Curr Opin Crit Care 14: 666-672, 2008.

4. Heckbert SR, Vedder NB, Hoffman W, Winn RK, Hudson LD, Jurkovich GJ, Copass MK, Harlan JM, Rice CL and Maier RV: Outcome after hemorrhagic shock in trauma patients. J Trauma 45: 545-549, 1998.

5. Hurt RT, Zakaria R, Matheson PJ, Cobb ME, Parker JR and Garrison RN: Hemorrhage-induced hepatic injury and hypoperfusion can be prevented by direct peritoneal resuscitation. J Gastrointest Surg 13: 587-594, 2009.

6. Kan WH, Hsu JT, Schwacha MG, Choudhry MA, Raju R, Bland KI and Chaudry IH: Selective inhibition of iNOS attenuates trauma-hemorrhage/resuscitation-induced hepatic injury. J Appl Physiol 1985 105: 1076-1082, 2008.
7. Matsutani T, Kang SC, Miyashita M, Sasajima K, Choudhry MA, Bland KI and Chaudry IH: Liver cytokine production and ICAM-1 expression following bone fracture, tissue trauma, and hemorrhage in middle-aged mice. Am J Physiol Gastrointest Liver Physiol 292: G268-G274, 2007.

8. Botha AJ, Moore FA, Moore EE, Kim FJ, Banerjee A and Peterson VM: Post injury neutrophil priming and activation: an early vulnerable window. Surgery 118: 358-364, 1995.

9. Hogg JC: Neutrophil kinetics and lung injury. Physiol Rev 67: 1249-1295, 1987.

10. Kensler TW, Wakabayashi $\mathrm{N}$ and Biswal S: Cell survival responses to environmental stresses via the Keap1-Nrf2-ARE pathway. Annu Rev Pharmacol Toxicol 47: 89-116, 2007.

11. Cho HY, Reddy SP, Yamamoto M and Kleeberger SR: The transcription factor NRF2 protects against pulmonary fibrosis. FASEB J 18: 1258-1260, 2004.

12. Rangasamy T, Guo J, Mitzner WA, Roman J, Singh A, Fryer AD, Yamamoto M, Kensler TW, Tuder RM, Georas SN and Biswal S: Disruption of Nrf2 enhances susceptibility to severe airway inflammation and asthma in mice. J Exp Med 202: 47-59, 2005.

13. Rangasamy T, Cho CY, Thimmulappa RK, Zhen L, Srisuma SS, Kensler TW, Yamamoto M, Petrache I, Tuder RM and Biswal S: Genetic ablation of Nrf2 enhances susceptibility to cigarette smokeinduced emphysema in mice. J Clin Invest 114: 1248-1259, 2004.

14. Osburn WO, Karim B, Dolan PM, Liu G, Yamamoto M, Huso DL and Kensler TW: Increased colonic inflammatory injury and formation of aberrant crypt foci in Nrf2-deficient mice upon dextran sulfate treatment. Int J Cancer 121: 1883-1891, 2007.

15. Thimmulappa RK, Lee H, Rangasamy T, Reddy SP, Yamamoto M, Kensler TW and Biswal S: Nrf2 is a critical regulator of the innate immune response and survival during experimental sepsis. J Clin Invest 116: 984-995, 2006.

16. Malhotra D, Thimmulappa R, Navas-Acien A, Sandford A, Elliott M, Singh A, Chen L, Zhuang X, Hogg J, Pare P, et al: Decline in NRF2-regulated antioxidants in chronic obstructive pulmonary disease lungs due to loss of its positive regulator, DJ-1. Am J Respir Crit Care Med 178: 592-604, 2008.

17. Kumawat M, Sharma TK, Singh I, Singh N, Ghalaut VS, Vardey SK and Shankar V: Antioxidant enzymes and lipid peroxidation in type 2 diabetes mellitus patients with and without nephropathy. N Am J Med Sci 5: 213-219, 2013.

18. Itoh K, Chiba T, Takahashi S, Ishii T, Igarashi K, Katoh Y, Oyake T, Hayashi N, Satoh K, Hatayama I, et al: An Nrf2/small Maf heterodimer mediates the induction of phase II detoxifying enzyme genes through antioxidant response elements. Biochem Biophys Res Commun 236: 313-322, 1997.

19. Hsieh CH, Frink M, Hsieh YC, Kan WH, Hsu JT, Schwacha MG, Choudhry MA and Chaudry IH: The role of MIP-1 alpha in the development of systemic inflammatory response and organ injury following trauma hemorrhage. J Immunol 181: 2806-2812, 2008.

20. Gray KD, Simovic MO, Blackwell TS, Christman JW, May AK, Parman KS, Chapman WC and Stain SC: Activation of nuclear factor kappa B and severe hepatic necrosis may mediate systemic inflammation in choline-deficient/ethionine-supplemented diet-induced pancreatitis. Pancreas 33: 260-267, 2006.

21. Vnukov VV, Gutsenko OI, Milutina NP, Kornienko IV Ananyan AA, Danilenko AO, Panina SB, Plotnikov AA and Makarenko MS: Influence of SkQ1 on expression of Nrf2 gene, ARE-controlled genes of antioxidant enzymes and their activity in rat blood leukocytes. Biochemistry (Mosc) 80: 1598-1605, 2015.

22. Morzadec C, Macoch M, Sparfel L, Kerdine-Römer S, Fardel O, Vernhet L: Nrf2 expression and activity in human T lymphocytes: stimulation by $\mathrm{T}$ cell receptor activation and priming by inorganic arsenic and tert-butylhydroquinone. Free Radic Biol Med 71: 133-145, 2014.

23. Rao DA and Pober JS: Endothelial injury, alarmins, and allograft rejection. Crit Rev Immunol 28: 229-248, 2008.

24. Yang D, Tewary P, de la Rosa G, Wei F and Oppenheim JJ: The alarmin functions of high-mobility group proteins. Biochim Biophys Acta 1799: 157-163, 2010.

25. Reynolds PS, Barbee RW, Skaflen MD and Ward KR: Low-volume resuscitation cocktail extends survival after severe hemorrhagic shock. Shock 28: 45-52, 2007.

26. Barbee RW, Reynolds PS and Ward KR: Assessing shock resuscitation strategies by oxygen debt repayment. Shock 33: 113-122, 2010.

27. Gutierrez G, Reines HD and Wulf-Gutierrez ME: Clinical review: hemorrhagic shock. Crit Care 8: 373-381, 2004.

28. Fan J, Frey RS and Malik AB: TLR4 signaling induces TLR2 expression in endothelial cells via neutrophil NADPH oxidase. J Clin Invest 112: 1234-1243, 2003. 
29. Imai Y, Kuba K, Neely GG, Yaghubian-Malhami R, Perkmann T, van Loo G, Ermolaeva M, Veldhuizen R, Leung YH, Wang H, et al: Identification of oxidative stress and Toll-like receptor 4 signaling as a key pathway of acute lung injury. Cell 133: 235-249, 2008.

30. Gill R, Ruan X, Menzel CL, Namkoong S, Loughran P, Hackam DJ and Billiar TR: Systemic inflammation and liver injury following hemorrhagic shock and peripheral tissue trauma involve functiona TLR9 signaling on bone marrow-derived cells and parenchymal cells. Shock 35: 164-170, 2011.

31. Song Z, Zhao X, Liu M, Jin H, Wang L, Hou M and Gao Y: Recombinant human brain natriuretic peptide attenuates trauma-l haemorrhagic shock-induced acute lung injury through inhibiting oxidative stress and the NF- $\kappa \mathrm{B}$-dependent inflammatory/MMP-9 pathway. Int J Exp Pathol 96: 406-413, 2015.

32. Bedard K and Krause KH: The NOX family of ROS-generating NADPH oxidases: physiology and pathophysiology. Physiol Rev 87: 245-313, 2007.

33. Krause KH and Bedard K: NOX enzymes in immuno-inflammatory pathologies. Semin Immunopathol 30: 193-194, 2008.

34. Lorne E, Zmijewski JW, Zhao X, Liu G, Tsuruta Y, Park YJ, Dupont $\mathrm{H}$ and Abraham E: Role of extracellular superoxide in neutrophil activation: interactions between xanthine oxidase and TLR4 induce proinflammatory cytokine production. Am J Physiol Cell Physiol 294: C985-C993, 2008.

35. Mitra S and Abraham E: Participation of superoxide in neutrophil activation and cytokine production. Biochim Biophys Acta 1762: 732-741, 2006.

36. Liu LM and Dubick MA: Hemorrhagic shock-induced vascular hyporeactivity in the rat: relationship to gene expression of nitric oxide synthase, endothelin-1, and select cytokines in corresponding organs. J Surg Res 125: 128-136, 2005.
37. Ertel W, Keel M, Neidhardt R, Steckholzer U, Kremer JP, Ungethuem $U$ and Trentz O: Inhibition of the defense system stimulating interleukin-12 interferon-gamma pathway during critical illness. Blood 89: 1612-1620, 1997.

38. Wang H, Bloom O, Zhang M, Vishnubhakat JM, Ombrellino M, Che J, Frazier A, Yang H, Ivanova S, Borovikova L, et al: HMG-1 as a late mediator of endotoxin lethality in mice. Science 285: 248-251, 1999

39. Yang H, Ochani M, Li J, Qiang X, Tanovic M, Harris HE, Susarla SM, Ulloa L, Wang H, DiRaimo R, et al: Reversing established sepsis with antagonists of endogenous high-mobility group box 1. Proc Natl Acad Sci USA 101: 296-301, 2004.

40. Tsung A, Sahai R, Tanaka H, Nakao A, Fink MP, Lotze MT, Yang H, Li J, Tracey KJ, Geller DA and Billiar TR: The nuclear factor HMGB1 mediates hepatic injury after murine liver ischemia-reperfusion. J Exp Med 201: 1135-1143, 2005.

41. Kim JY, Park JS, Strassheim D, Douglas I, Diaz del Valle F Asehnoune K, Mitra S, Kwak SH, Yamada S, Maruyama I, et al: HMGB1 contributes to the development of acute lung injury after hemorrhage. Am J Physiol Lung Cell Mol Physiol 288: L958-L965, 2005.

42. Andersson U, Erlandsson-Harris H, Yang H and Tracey KJ: HMGB1 as a DNA-binding cytokine. J Leukoc Biol 72: 1084-1091, 2002

43. Ombrellino M, Wang H, Ajemian MS, Talhouk A, Scher LA, Friedman SG and Tracey KJ: Increased serum concentrations of high-mobility-group protein 1 in haemorrhagic shock. Lancet 354: 1446-1447, 1999. 